\title{
Optimal Distortion-Power Tradeoffs in Sensor Networks: Gauss-Markov Random Processes
}

\author{
Nan Liu Sennur Ulukus \\ Department of Electrical and Computer Engineering \\ University of Maryland, College Park, MD 20742 \\ nkancy@umd.edu_ulukus@umd.edu
}

\begin{abstract}
We investigate the optimal performance of dense sensor networks by studying the joint source-channel coding problem. The overall goal of the sensor network is to take measurements from an underlying random process, code and transmit those measurement samples to a collector node in a cooperative multiple access channel with feedback, and reconstruct the entire random process at the collector node. We provide lower and upper bounds for the minimum achievable expected distortion when the underlying random process is stationary and Gaussian. In the case where the random process is also Markovian, we evaluate the lower and upper bounds explicitly and show that they are of the same order for a wide range of sum power constraints. Thus, for a Gauss-Markov random process, under these sum power constraints, we determine the achievability scheme that is order-optimal, and express the minimum achievable expected distortion as a function of the sum power constraint.
\end{abstract}

\section{INTRODUCTION}

With the recent advances in the hardware technology, small cheap nodes with sensing, computing and communication capabilities have become available. In practical applications, it is possible to deploy a large number of these nodes to sense the environment. In this paper, we investigate the optimal performance of a dense sensor network by studying the joint sourcechannel coding problem. The sensor network is composed of $N$ sensors, where $N$ is very large, and a single collector node. The overall goal of the sensor network is to take measurements from an underlying random process $S(t), 0 \leq t \leq T_{0}$, code and transmit those measured samples to a collector node in a cooperative multiple access channel with feedback, and reconstruct the entire random process at the collector node. We investigate the minimum achievable expected distortion and the corresponding achievability scheme when the underlying random process is Gaussian and the communication channel is a cooperative Gaussian multiple access channel with feedback.

Following the seminal paper of Gupta and Kumar [1], which showed that multi-hop wireless ad-hoc networks, where users transmit independent data and utilize single-user coding, decoding and forwarding techniques, do not scale up, Scaglione and Servetto [2] investigated the scalability of the sensor networks. Sensor networks, where the observed data is correlated, may scale up for two reasons: first, the correlation among the sampled data increases with the increasing number

This work was supported by NSF Grants CCR 03-11311, CCF 04-47613 and CCF 05-14846; and ARL/CTA Grant DAAD 19-01-2-0011. of nodes and hence, the amount of information the network needs to carry does not increase as fast as in ad-hoc wireless networks; and second, correlated data facilitates cooperation, and may increase the information carrying capacity of the network. The goal of the sensor network in [2] was that each sensor reconstructs the data measured by all of the sensors using sensor broadcasting. In this paper, we focus on the case where the reconstruction is required only at the collector node. Also, in this paper, the task is not the reconstruction of the data the sensors measured, but the reconstruction of the underlying random process.

Gastpar and Vetterli [3] studied the case where the sensors observe a noisy version of a linear combination of $L$ Gaussian random variables with equal variances, code and transmit those observations to a collector node, and the collector node reconstructs the $L$ random variables. In [3], the expected distortion achieved by applying separation-based approaches was shown to be exponentially worse than the lower bound on the minimum expected distortion. In this paper, we study the case where the data of interest at the collector node is not a finite number of random variables, but a random process, which, using Karhunen-Loeve expansion, can be shown to be equivalent to a set of infinitely many random variables with varying variances. We assume that the sensors are able to take noiseless samples, but that each sensor observes only its own sample. Our upper bound is also developed by using a separation-based approach, but it is shown to be of the same order as the lower bound, for a wide range of power constraints for a Gauss-Markov random process.

El Gamal [4] studied the capacity of dense sensor networks and found that all spatially band-limited Gaussian processes can be estimated at the collector node, subject to any non-zero constraint on the mean squared distortion. In this paper, we study the minimum achievable expected distortion for spacelimited, and thus, not band-limited, random processes, and we show that the minimum achievable expected distortion decreases to zero as the number of nodes increases, unless the sum power constraint is unusually small.

We first provide lower and upper bounds for the minimum achievable expected distortion for arbitrary stationary Gaussian random processes. Then, we focus on the case where the Gaussian random process is also Markovian, evaluate the lower and upper bounds explicitly, and show that they are of the same order, for a wide range of power constraints. 
Thus, for a Gauss-Markov random process, under a wide range of power constraints, we determine an order-optimal achievability scheme, and identify the minimum achievable expected distortion. Our order-optimal achievability scheme is separation-based. It is well-known [5], [6] that in multi-user channels with correlated sources, the source-channel separation principle does not hold in general, and separation-based achievability schemes may be strictly suboptimal. However, in this instance, where we have a multi-user channel with correlated sources, for a wide range of power constraints, we show that a separation-based achievability scheme is orderoptimal, when the number of nodes goes to infinity.

The results of this paper provide insights for the design of large sensor networks that aim at reconstructing the underlying random process at a collector node. Our results provide the order-optimal scheme for the operation of the sensor nodes, the number of nodes needed to be deployed and the power constraint needed to be employed, in order to achieve a certain overall distortion.

Although, we constrain ourselves to Gauss-Markov processes in this paper, we believe that our methods can be extended to more general Gaussian random processes.

\section{SYSTEM MODEL}

The collector node wishes to reconstruct a random process $S(t)$, for $0 \leq t \leq T_{0}$, where $t$ denotes the spatial position; $S(t)$ is assumed to be Gaussian and stationary with autocorrelation function $C(\tau)$. The $N$ sensor nodes are placed at positions $0=t_{1} \leq t_{2} \leq \cdots \leq t_{N}=T_{0}$, and observe samples $\mathbf{S}_{N}=\left(S\left(t_{1}\right), S\left(t_{2}\right), \cdots, S\left(t_{N}\right)\right)$. For simplicity and to avoid irregular cases, we assume that the sensors are equally spaced. The distortion measure is the squared error,

$$
d(s(t), \hat{s}(t))=\frac{1}{T_{0}} \int_{0}^{T_{0}}(s(t)-\hat{s}(t))^{2} d t
$$

Each sensor node and the collector node, denoted as node 0 , is equipped with one transmit and one receive antenna. At any time instant, let $X_{i}$ and $Y_{i}$ denote the signals transmitted by and received at, node $i$, and let $h_{j i}$ denote the channel gain from node $j$ to node $i$. Then, the received signal at node $i$ can be written as,

$$
Y_{i}=\sum_{j=0, j \neq i}^{N} h_{j i} X_{j}+Z_{i}, \quad i=0,1,2, \cdots, N
$$

where $\left\{Z_{i}\right\}_{i=0}^{N}$ is a vector of $N+1$ independent and identically distributed, zero-mean, unit-variance Gaussian random variables. Therefore, the channel model of the network is such that all nodes hear a linear combination of the signals transmitted by all other nodes at that time instant. We assume that $h_{i j}$ is determined by the distance between nodes $i$ and $j$, denoted as $d_{i j}$, as $h_{i j}=d_{i j}^{-\alpha / 2}$ for $i, j=0,1,2, \cdots, N$, and $\alpha$ is the path-loss exponent, which is typically between 2 and 6 [7]. For simplicity, we assume that the collector node is at an equal distance away from all of the sensor nodes, i.e., $h_{i 0}=h$, for $i=1,2, \cdots, N$, where $h$ is some constant, independent of $N$. The results can be generalized straightforwardly to the case where $h_{i 0}$ are non-identical constants.

We assume that all sensors share a sum power constraint of $P(N)$ which is a function of $N$. For the discussion of distortion-power tradeoffs of the Gauss-Markov processes, we divide $P(N)$ into five regions.

- Very large: $P(N)$ is larger than $\frac{e^{N}}{N}$.

- Large: $P(N)$ is between $\frac{e^{N^{1 / 3}}}{N}$ and $\frac{e^{N}}{N}$.

- Medium: $P(N)$ is between $N^{-\frac{1}{1+\frac{1}{\alpha}}}$ and $\frac{e^{N^{1 / 3}}}{N}$.

- Small: $P(N)$ is between $N^{-1}$ and $N^{-\frac{1}{1+\frac{1}{\alpha}}}$.

- Very small: $P(N)$ is no larger than $N^{-1}$.

The reason why we divide $P(N)$ into these five regions will be apparent in Sections IV and V. The two most interesting cases for the sum power constraint are $P(N)=N P_{\text {ind }}$ where each sensor has its individual power constraint $P_{\text {ind }}$, and $P(N)=$ $P_{\text {tot }}$ where all sensors share a constant total power constraint $P_{\text {tot }}$. Both of these two cases lie in the medium sum power constraint region. Our goal is to determine the scheme that achieves the minimum expected distortion $D^{N}$ at the collector node for a given total transmit power constraint $P(N)$, and also to determine the rate at which this distortion goes to zero as a function of the number of sensor nodes and the power constraint.

In this paper, we seek to understand the behavior of the minimum achievable expected distortion when the number of sensor nodes is very large. We introduce the big-O and big$\Theta$ notations. We say that $f$ is $\mathrm{O}(g)$, if there exist constants $c$ and $k$, such that $|f(N)| \leq c|g(N)|$ for all $N>k$; we say that $f$ is $\Theta(g)$, if there exist constants $c_{1}, c_{2}$ and $k$ such that $c_{1}|g(N)| \leq|f(N)| \leq c_{2}|g(N)|$ for all $N>k$. All logarithms are base $e$. Due to space limitations, all proofs are omitted here and can be found in [8].

\section{The GAUSS-MARKOV PROCESS}

A Gauss-Markov process, also known as the OrnsteinUhlenbeck process [9], [10], is defined as a random process that is stationary, Gaussian, Markovian, and continuous in probability. It is known that the autocorrelation function of this process is [11]-[13]

$$
C(\tau)=\frac{\sigma^{2}}{2 \eta} e^{-\eta|\tau|}
$$

The Karhunen-Loeve expansion [14] of the Gauss-Markov process yields the eigenfunctions $\left\{\phi_{k}(t)\right\}_{k=0}^{\infty}$

$\phi_{k}(t)=b_{k}\left(\cos \sqrt{\frac{\sigma^{2}}{\lambda_{k}}-\eta^{2}} t+\frac{\eta}{\sqrt{\frac{\sigma^{2}}{\lambda_{k}}-\eta^{2}}} \sin \sqrt{\frac{\sigma^{2}}{\lambda_{k}}-\eta^{2}} t\right)$

where $\left\{\lambda_{k}\right\}_{k=0}^{\infty}$ are the corresponding eigenvalues and $b_{k}$ are positive constants chosen such that the eigenfunctions $\phi_{k}(t)$ have unit energy. Even though it is not possible to express $\left\{\lambda_{k}\right\}_{k=0}^{\infty}$ in closed form, they can be bounded as

$$
\lambda_{k}^{\prime} \leq \lambda_{k} \leq \lambda_{k}^{\prime \prime}
$$


where $\left\{\lambda_{k}^{\prime}\right\}_{k=1}^{\infty}$ is defined as

$$
\lambda_{k}^{\prime}= \begin{cases}\frac{\sigma^{2} T_{0}^{2}}{\left(k+\frac{1}{2}\right)^{2} \pi^{2}+\eta^{2} T_{0}^{2}}, & k \leq K_{0} \\ \frac{\sigma^{2} T_{0}^{2}}{(k+1)^{2} \pi^{2}}, & k>K_{0}\end{cases}
$$

with $K_{0}=\left\lfloor\frac{\eta^{2} T_{0}^{2}}{\pi^{2}}-\frac{3}{4}\right\rfloor$ and $\lfloor x\rfloor$ is the largest integer smaller than or equal to $x$; also, $K_{0}$ may be negative, in which case, the first line in (6) should be disregarded. $\left\{\lambda_{k}^{\prime \prime}\right\}_{k=1}^{\infty}$ is defined as

$$
\lambda_{k}^{\prime \prime}= \begin{cases}\frac{\sigma^{2}}{\eta^{2}}, & k \leq 1 \\ \frac{\sigma^{2} T_{0}^{2}}{(k-1)^{2} \pi^{2}}, & k>1\end{cases}
$$

Rate-distortion functions are easier to calculate with $\left\{\lambda_{k}^{\prime}\right\}_{k=0}^{\infty}$ and $\left\{\lambda_{k}^{\prime \prime}\right\}_{k=0}^{\infty}$, and the two sequences will be used in place of $\left\{\lambda_{k}\right\}_{k=0}^{\infty}$ to develop lower and upper bounds on the minimum achievable expected distortion.

\section{A Lower Bound on the Minimum Achievable EXPECTED DISTORTION}

\section{A. Arbitrary Stationary Gaussian Random Processes}

Let $D^{N}$ be the minimum achievable expected distortion at the collector node for a given total transmit power constraint $P(N)$. In this section, we will develop two lower bounds on $D^{N}$. We obtain our first lower bound by assuming that the communication links from the sensor nodes to the collector node are noise and interference free. Let $D_{s}^{N}$ be the MMSE (minimum mean squared error) when the collector node estimates the underlying random process by using the exact values of all of the samples taken by the sensors. Then, it is straightforward to see that,

$$
D^{N} \geq D_{s}^{N}
$$

Since the random process is Gaussian, calculating $D_{s}^{N}$ is a Gaussian MMSE estimation problem. It suffices to consider the linear MMSE estimator and the resulting expected distortion is

$$
D_{s}^{N}=\frac{1}{T_{0}} \int_{0}^{T_{0}}\left(C(0)-\boldsymbol{\rho}_{N}^{T}(t) \Sigma_{N}^{-1} \boldsymbol{\rho}_{N}(t)\right) d t
$$

where

$$
\boldsymbol{\rho}_{N}(t)=\left[\begin{array}{llll}
C\left(t-t_{1}\right) & C\left(t-t_{2}\right) & \cdots & C\left(t-t_{N}\right)
\end{array}\right]^{T}
$$

and

$$
\begin{aligned}
\Sigma_{N} & =E\left[\mathbf{S}_{N} \mathbf{S}_{N}^{T}\right] \\
& =\left[\begin{array}{cccc}
C(0) & C\left(t_{2}-t_{1}\right) & \cdots & C\left(t_{N}-t_{1}\right) \\
C\left(t_{2}-t_{1}\right) & C(0) & \cdots & C\left(t_{N}-t_{2}\right) \\
\vdots & \vdots & \vdots & \vdots \\
C\left(t_{N}-t_{1}\right) & C\left(t_{N}-t_{2}\right) & \cdots & C(0)
\end{array}\right]
\end{aligned}
$$

We obtain our second lower bound by assuming that all of the sensors know the random process exactly, and, the sensor network forms an $N$-transmit 1-receive antenna pointto-point system to transmit the random process to the collector node. Let $C_{u}^{N}$ be the capacity of this point-to-point system and $D_{p}(R)$ be the distortion-rate function of the random process $S(t)$ [15]. In this point-to-point system, the separation principle holds and feedback does not increase the capacity, and therefore

$$
D^{N} \geq D_{p}\left(C_{u}^{N}\right)
$$

To evaluate $D_{p}\left(C_{u}^{N}\right)$, we first find the rate distortion function, $R(D)$, of $S(t)[15$, Section 4.5] as,

$$
R(\theta)=\sum_{k=0}^{\infty} \max \left(0, \frac{1}{2} \log \left(\frac{\lambda_{k}}{\theta}\right)\right)
$$

and

$$
D(\theta)=T_{0}^{-1} \sum_{k=0}^{\infty} \min \left(\theta, \lambda_{k}\right)
$$

It can be seen that the function $R(\theta)$ is a strictly decreasing function of $\theta$ when $\theta \leq \lambda_{0}$. Hence, in this region, the inverse function of $R(\theta)$ exists, which we will call $\theta(R), R \geq 0$. Next, we find $C_{u}^{N}$, the capacity of the $N$-transmit 1-receive antenna point-to-point system [16] as,

$$
C_{u}^{N}=\frac{1}{2} \log \left(1+h^{2} N P(N)\right)
$$

Then, we have

$$
D_{p}\left(C_{u}^{N}\right)=D\left(\theta\left(C_{u}^{N}\right)\right)
$$

By combining the two lower bounds described above, we see that, for arbitrary stationary Gaussian random processes, a lower bound on the minimum achievable expected distortion is

$$
D_{l}^{N}=\max \left(D_{s}^{N}, D_{p}\left(C_{u}^{N}\right)\right)
$$

\section{B. The Gauss-Markov Process}

We note that $D_{s}^{N}$ and $D_{p}\left(C_{u}^{N}\right)$ in (17) both depend on the autocorrelation function $C(\tau)$. Unless we put more structure on $C(\tau)$, it seems difficult to continue with an exact evaluation. Hence, we constrain ourselves to a special class of Gaussian random processes, the Gauss-Markov random processes, whose autocorrelation function is given in (3), in order to continue with our analysis of the distortion.

First, we evaluate $D_{s}^{N}$. Using (3) and the Markovian property of $S(t)$, it is straightforward to show that [8],

$$
D_{s}^{N}=\Theta\left(N^{-1}\right)
$$

Hence, for the Gauss-Markov process when the random process is estimated from its samples, the estimation error decays as $N^{-1}$.

Next, we evaluate $D_{p}\left(C_{u}^{N}\right)$ for the Gauss-Markov process. Let $D_{p}^{\prime}\left(C_{u}^{N}\right)$ be the distortion obtained from (16) when $\left\{\lambda_{k}\right\}_{k=0}^{\infty}$ in (13) and (14) are replaced by $\left\{\lambda_{k}^{\prime}\right\}_{k=0}^{\infty}$ which we defined in (5) and (6). Then,

$$
D_{p}^{\prime}\left(C_{u}^{N}\right) \leq D_{p}\left(C_{u}^{N}\right)
$$


because $\lambda_{k}^{\prime} \leq \lambda_{k}$ for all $k$, and it is more difficult to estimate a sequence of random variables each with a larger variance. Since we seek a lower bound on the minimum achievable expected distortion, the evaluation of $D_{p}^{\prime}\left(C_{u}^{N}\right)$ suffices. Hence, for the rest of this section, we concentrate on the evaluation of $R(\theta)$ and $D(\theta)$ given in (13) and (14), respectively, for $\left\{\lambda_{k}^{\prime}\right\}_{k=0}^{\infty}$.

We will divide our discussion into two separate cases based on the sum power constraint. For the first case, $P(N)$ is such that

$$
\lim _{N \rightarrow \infty}(N P(N))^{-1}=0
$$

is satisfied. This includes all sum power constraint regions defined in Section II except the very small sum power constraint. The cases where $P(N)=N P_{\text {ind }}$ and $P(N)=P_{\text {tot }}$ are included in $P(N)$ satisfying (20). Note from (15) that, in this case, $C_{u}^{N}$ increases monotonically in $N$. Since we are interested in the number $\theta\left(C_{u}^{N}\right)$, we consider the region when $R$ is very large for the function $\theta(R)$.

Lemma 1 For large enough $R$, we have

$$
\theta(R) \geq\left(\frac{\sigma T_{0}}{2 \pi R}\right)^{2}
$$

We bound $D(\theta)$ for small enough $\theta$ in the next lemma.

Lemma 2 For small enough $\theta$, we have

$$
D(\theta) \geq \frac{\sigma}{\pi} \sqrt{\theta}
$$

We are now ready to calculate the distortion. When $N$ is large enough, using (15), (21) and (22), we have

$$
D_{p}^{\prime}\left(C_{u}^{N}\right) \geq \Theta\left((\log (N P(N)))^{-1}\right)
$$

We conclude, based on (17), (18), (19) and (23), that when the sum power constraint $P(N)$ satisfies (20), a lower bound on the minimum achievable expected distortion is

$$
D^{N} \geq \Theta\left(\max \left(N^{-1},(\log (N P(N)))^{-1}\right)\right)
$$

For the second case, $P(N)$ is such that (20) is not satisfied. $C_{u}^{N}$ is either a constant independent of $N$ or goes to zero as $N$ goes to infinity. Examining (13), we see that $\theta\left(C_{u}^{N}\right)$ is bounded below by a constant independent of $N$, and hence, $D_{p}^{\prime}\left(C_{u}^{N}\right)$ is a constant and does not go to zero as $N$ increases.

Therefore, for all possible power constraints $P(N)$, a lower bound on the minimum achievable expected distortion is

$$
D^{N} \geq \Theta\left(\max \left(N^{-1}, \min \left((\log (N P(N)))^{-1}, 1\right)\right)\right)
$$

which can also be expressed "order-wise" as

$$
\begin{cases}N^{-1} & \text { if } \lim _{N \rightarrow \infty} \frac{e^{N}}{N P(N)}=0 \\ 1 & \text { if } \lim _{N \rightarrow \infty} N P(N)=0 \\ (\log (N P(N)))^{-1} & \text { otherwise }\end{cases}
$$

The first case in (26) corresponds to the very large sum power constraint defined in Section II. This is the scenario where the sum power constraint grows almost exponentially with the number of nodes. The transmission power is so large that the communication channels between the sensors and the collector node are as if they are perfect, and we are left with the "unavoidable" distortion of $N^{-1}$ which we have in reconstructing the random process from the "perfect" knowledge of its samples. Even though this provides the best performance among all three cases, it is impractical since sensor nodes are low energy devices and it is often difficult, if not impossible, to replenish their batteries.

The second case in (26) corresponds to the very small sum power constraint defined in Section II. The transmission power is so low that the communication channels between the sensors and the collector node are as if they do not exist. The estimation error is on the order of 1 , which is equivalent to the collector node blindly estimating $S(t)=0$ for all $t \in\left[0, T_{0}\right]$. Even though the consumed power $P(N)$ is very low in this case, the performance of the sensor network is unacceptable; even the lower bound on the minimum achievable expected distortion does not decrease to zero with the increasing number of nodes.

Hence, the meaningful sum power constraints for the sensor nodes should be in the "otherwise" case in (26), which includes the large, medium and small sum power constraints defined in Section II. The corresponding lower bound on the minimum achievable expected distortion as a function of the power constraint is

$$
D^{N} \geq \Theta\left((\log (N P(N)))^{-1}\right)
$$

The two practically meaningful cases of $P(N)=N P_{\text {ind }}$ and $P(N)=P_{\text {tot }}$ are in this "otherwise" case. In both of these cases, the lower bound on the minimum achievable expected distortion decays to zero at the rate of $(\log N)^{-1}$.

\section{An Upper Bound on the Minimum Achievable EXPECTED DISTORTION}

\section{A. Arbitrary Stationary Gaussian Random Processes}

Any distortion found by using any achievability scheme will serve as an upper bound for the minimum achievable expected distortion. We consider the following separationbased achievable scheme: First, we perform distributed ratedistortion coding at all sensor nodes using [17, Theorem 1]. After obtaining the indices of the rate-distortion codes, we transmit the indices as independent messages using the antenna sharing method introduced in [4]. The distortion obtained using this scheme will be denoted as $D_{u}^{N}$.

We apply [17, Theorem 1], generalized to $N$ sensor nodes in [18, Theorem 1], to obtain an achievable rate-distortion point. We will consider the case when all sensor nodes transmit their data at identical rates, and this rate is determined by the ratio of the sum rate and $N$. We have the following theorem.

Theorem 1 The following sum rate and distortion are achiev- 
able,

$D_{a}^{N}\left(\theta^{\prime}\right)=C(0)-\frac{1}{N-1} \int_{0}^{T_{0}} \boldsymbol{\rho}_{N}^{T}(t)\left(\Sigma_{N}^{\prime}+\theta^{\prime} I\right)^{-1} \boldsymbol{\rho}_{N}(t) d t$

$R_{a}^{N}\left(\theta^{\prime}\right)=\sum_{k=0}^{N-1} \frac{1}{2} \log \left(1+\frac{\mu_{k}^{(N)^{\prime}}}{\theta^{\prime}}\right)$

where $\Sigma_{N}^{\prime}=\frac{T_{0}}{N-1} \Sigma_{N}$ and $\mu_{0}^{(N)^{\prime}}, \mu_{1}^{(N)^{\prime}}, \cdots, \mu_{N-1}^{(N)^{\prime}}$ are the eigenvalues of $\Sigma_{N}^{\prime}$.

We further evaluate $D_{a}^{N}\left(\theta^{\prime}\right)$ in the next lemma.

Lemma 3 For all stationary Gaussian random processes whose autocorrelation functions satisfy the Lipschitz condition in the interval $\left[-T_{0}, T_{0}\right]$, and have finite right derivatives at $\tau=0$, we have

$$
D_{a}^{N}\left(\theta^{\prime}\right)=O\left(\max \left(N^{-1}, \frac{1}{T_{0}} \sum_{k=1}^{N}\left(\frac{1}{\theta^{\prime}}+\frac{1}{\mu_{k}^{(N)^{\prime}}}\right)^{-1}\right)\right)
$$

Lemma 3 tells us that the expected distortion achieved by using the separation-based scheme is upper bounded by the maximum of two types of distortion. The first distortion is of size $N^{-1}$ and the size of the second distortion depends on the achievable rate of the channel through $\theta^{\prime}$. We define the second distortion as

$$
D_{b}^{N}\left(\theta^{\prime}\right)=\frac{1}{T_{0}} \sum_{k=1}^{N}\left(\frac{1}{\theta^{\prime}}+\frac{1}{\mu_{k}^{(N)^{\prime}}}\right)^{-1}
$$

Now, we determine an achievable rate for the communication channel from the sensor nodes to the collector node. The channel in its nature is a multiple access channel with potential cooperation between the transmitters and feedback from the collector node. The capacity region for this channel is not known. We get an achievable sum rate for this channel by using the idea presented in [4]. The following theorem is a generalization of $[4$, Theorem 1] from a constant power constraint to a more general power constraint.

Theorem 2 When the sum power constraint $P(N)$ and the path-loss exponent $\alpha$ satisfy

$$
\lim _{N \rightarrow \infty} \frac{1}{N P(N)^{1+\frac{1}{\alpha}}}=0
$$

the following rate is achievable

$$
C_{a}^{N}=\beta \log (N P(N))
$$

where $\beta$ is a positive constant defined as

$$
\beta=\frac{1+\frac{1}{\alpha} \lim _{N \rightarrow \infty} \frac{\log P(N)}{\log (N P(N))}}{4\left(1+\frac{1}{\alpha}\right)}
$$

otherwise, $C_{a}^{N}$ approaches a non-negative constant as $N \rightarrow$ $\infty$.
Theorem 2 shows that when the sum power constraint is very large, large or medium, as defined in Section II, the achievable rate increases with $N$. Otherwise, the achievable rate is either a positive constant or decreases to zero, which will result in poor estimation performance at the collector node.

The function $R_{a}^{N}\left(\theta^{\prime}\right)$ is a strictly decreasing function of $\theta^{\prime}$, thus, the inverse function exists, which we will denote as $\theta_{a}^{N}(R)$. Hence, to find $D_{u}^{N}$, we first find $\theta_{a}^{N}\left(C_{a}^{N}\right)$, and then,

$$
D_{u}^{N}=D_{a}^{N}\left(\theta_{a}^{N}\left(C_{a}^{N}\right)\right)
$$

We will perform this calculation when the underlying random process is Gauss-Markov.

\section{B. The Gauss-Markov Process}

The autocorrelation function of the Gauss-Markov process given in (3) satisfies the conditions of Lemma 3. Hence, (30) is valid, and

$$
D_{u}^{N}=O\left(\max \left(N^{-1}, D_{b}\left(\theta_{a}^{N}\left(C_{a}^{N}\right)\right)\right)\right)
$$

It remains to evaluate $D_{b}^{N}\left(\theta_{a}^{N}\left(C_{a}^{N}\right)\right)$. We first define two sequences $\vartheta_{L}^{N}$ and $\vartheta_{U}^{N}$ which satisfy

$$
\lim _{N \rightarrow \infty} \frac{1}{\vartheta_{L}^{N} N^{2 / 3}}=0, \quad \lim _{N \rightarrow \infty} \vartheta_{U}^{N}=0
$$

Lemma 4 For large enough $N$ and $R$ in the interval of

$$
\left[\frac{8 \sigma T_{0}}{\pi \sqrt{\vartheta_{U}^{N}}}, \frac{\sigma T_{0}}{4 \pi \sqrt{\vartheta_{L}^{N}}}\right]
$$

we have

$$
\left(\frac{\sigma T_{0}}{4 \pi R}\right)^{2} \leq \theta_{a}^{N}(R) \leq\left(\frac{8 \sigma T_{0}}{\pi R}\right)^{2}
$$

Hence, for all $P(N)$ that satisfy

$$
\lim _{N \rightarrow \infty} \frac{N P(N)}{e^{N^{1 / 3}}}=0
$$

and (32), we have $C_{a}^{N}$ in the interval of (38) and our result of (39) is applicable.

Now we upper bound $D_{b}^{N}\left(\theta^{\prime}\right)$.

Lemma 5 For $\theta^{\prime} \in\left[\vartheta_{L}^{N}, \vartheta_{U}^{N}\right]$ for large enough $N$, we may upper bound $D_{b}^{N}\left(\theta^{\prime}\right)$ as

$$
D_{b}^{N}\left(\theta^{\prime}\right) \leq \frac{12 \sigma}{\pi} \sqrt{\theta^{\prime}}
$$

The proofs of Lemma 4 and 5 use the fact that $\mu_{k}^{(N)^{\prime}}$ converges to $\lambda_{k}$ when $N$ is large [2]. Since the convergence of $\mu_{k}^{(N)^{\prime}}$ to $\lambda_{k}$ is not uniform in $k$, the results of Lemma 4 and 5 are valid only when $P(N)$ satisfies (40).

Hence, when $P(N)$ is such that (40) and (32) are satisfied, using (39), (41) and the fact that when $R$ is in the interval of (38), $\theta_{a}^{N}(R)$ is in $\left[\vartheta_{L}^{N}, \vartheta_{U}^{N}\right]$, we have

$$
D_{b}^{N}\left(\theta_{a}^{N}\left(C_{a}^{N}\right)\right) \leq \Theta\left((\log (N P(N)))^{-1}\right)
$$


Therefore, from (36), an upper bound on the minimum achievable expected distortion is

$$
D_{u}^{N} \leq \Theta\left((\log (N P(N)))^{-1}\right)
$$

This upper bound on the minimum achievable expected distortion coincides with the lower bound described in the "otherwise" case in (26). However, it should be noted that, the "otherwise" case in (26) corresponds to the large, medium and small sum power constraints defined in Section II, whereas (40) and (32) are satisfied only for the medium sum power constraint.

\section{COMPARISON OF LOWER AND UPPER BOUNDS FOR GAUSS-MARKOV PROCESSES}

Now, we compare the upper bound in (43) and the lower bound in (26). In the very large and large sum power constraint regions, our methods do not apply, e.g. (43) is not valid, and we have not shown whether the lower and upper bounds meet. However, in this region $P(N)$ is larger than $\frac{e^{N^{1 / 3}}}{N}$, and this region is not of practical interest.

In the medium sum power constraint region, $P(N)$ is in the wide range of $N^{-\frac{1}{1+\frac{1}{\alpha}}}$ to $\frac{e^{N^{1 / 3}}}{N}$, and our lower and upper bounds do meet and the minimum achievable expected distortion is

$$
D^{N}=\Theta\left((\log (N P(N)))^{-1}\right)
$$

The order-optimal achievability scheme is a separation-based scheme, which uses distributed rate-distortion coding as described in [17] and optimal single-user channel coding with antenna sharing method as described in [4]. The practically interesting cases of $P(N)=N P_{\text {ind }}$ and $P(N)=P_{\text {tot }}$ fall into this region. In both of these cases, the minimum achievable expected distortion decreases to zero at the rate of

$$
(\log N)^{-1}
$$

Hence, the power constraint $P(N)=P_{\text {tot }}$ performs as well as $P(N)=N P_{\text {ind }}$ "order-wise", and therefore, in practice we may prefer to choose $P(N)=P_{\text {tot }}$.

In the small sum power constraint region where $P(N)$ ranges from $N^{-1}$ to $N^{-\frac{1}{1+\frac{1}{\alpha}}}$, our lower and upper bounds do not meet. The lower bound decreases to zero as $(\log N)^{-1}$ but the upper bound is a non-zero constant. The main discrepancy between the lower and upper bounds comes from the gap between the lower and upper bounds on the sum capacity, $C_{a}^{N}$ and $C_{u}^{N}$, for a cooperative multiple access channel with feedback. This region should be of practical interest because in this region, the sum power constraint is quite low, and yet the lower bound on the distortion is of the same order as any $P(N)$ which increases polynomially with $N$. Hence, from the results of the lower bound, it seems that this region potentially has good performance. However, our separation-based upper bound does not meet the lower bound, and whether the lower bound can be achieved remains an open problem.

In the very small sum power constraint region, $P(N)$ is less than $N^{-1}$, and our lower and upper bounds meet and the minimum achievable expected distortion is a constant that does not decrease to zero with increasing $N$. This case is not of practical interest because of the unacceptable distortion.

\section{CONCLUSION}

We investigate the performance of dense sensor networks by studying the joint source-channel coding problem. We provide lower and upper bounds for the minimum achievable expected distortion when the underlying random process is stationary and Gaussian. When the random process is also Markovian, we evaluate the lower and upper bounds, and show that they are both of order $(\log (N P(N)))^{-1}$ for a wide range of sum power constraints ranging from $N^{-\frac{1}{1+\frac{1}{\alpha}}}$ to $\frac{e^{N^{1 / 3}}}{N}$. In the most interesting cases when the sum power grows linearly with $N$ or is a constant, the minimum achievable expected distortion decreases to zero at the rate of $(\log N)^{-1}$. For a GaussMarkov process, under these power constraints, we have found that an order-optimal scheme is a separation-based scheme, that is composed of distributed rate-distortion coding [17] and antenna sharing method for cooperative multiple access channels [4]. We expect our results to be generalizable to more general classes of Gaussian random processes.

\section{REFERENCES}

[1] P. Gupta and P. R. Kumar. The capacity of wireless networks. IEEE Trans. on Information Theory, 46(2):388-404, March 2000.

[2] A. Scaglione and S. D. Servetto. On the interdependence of routing and data compression in multi-hop sensor networks. ACM/Kluwer Journal on Mobile Networks and Applications (MONET)—Selected (and revised) papers from ACM MobiCom, 2002. To appear.

[3] M. Gastpar and M. Vetterli. Power, spatio-temporal bandwidth, and distortion in large sensor networks. IEEE Journal on Selected Areas in Communications, 23(4):745-754, April 2005.

[4] H. El Gamal. On the scaling laws of dense wireless sensor networks: the data gathering channel. IEEE Trans. on Information Theory, 51(3):12291234, March 2005.

[5] T. M. Cover and J. A. Thomas. Elements of Information Theory. WileyInterscience, 1991.

[6] T. M. Cover, A. El Gamal, and M. Salehi. Multiple access channels with arbitrarily correlated sources. IEEE Trans. on Information Theory, 26(6):648 - 657, November 1980.

[7] T. S. Rappaport. Wireless communications: Principles and Practice. Prentice Hall, 1996.

[8] N. Liu and S. Ulukus. Optimal distortion-power tradeoffs in Gaussian sensor networks. To be submitted for journal publication.

[9] G. E. Uhlenbeck and L. S. Ornstein. On the theory of Brownian motion. Phys. Rev., 36, 1930.

[10] M. C. Wang and G. E. Uhlenbeck. On the theory of Brownian motion II. Rev. Modern Phys., 17, 1945.

[11] J. L. Doob. The Brownian movement and stochastic equations. Annals of Math., 43, 1942.

[12] L. Breiman. Probability. Addison-Wesley, 1968

[13] I. Karatzas and S. E. Shreve. Brownian motion and stochastic calculus. Springer-Verlag, 1988.

[14] A. Papoulis. Probability, Random Variables, and Stochastic Processes. McGraw-Hill, 1991.

[15] T. Berger. Rate Distortion Theory. Prentice Hall, 1971.

[16] I. E. Telatar. Capacity of multi-antenna Gaussian channels. European Trans. Telecommunications, 10:585-595, November 1999.

[17] T. J. Flynn and R. M. Gray. Encoding of correlated observations. IEEE Trans. on Information Theory, 33(6):773-787, November 1987.

[18] J. Chen, X. Zhang, T. Berger, and S. B. Wicker. An upper bound on the sum-rate distortion function and its corresponding rate allocation schemes for the CEO problem. IEEE Journal on Selected Areas of Communications, 22(6):977-987, August 2004. 